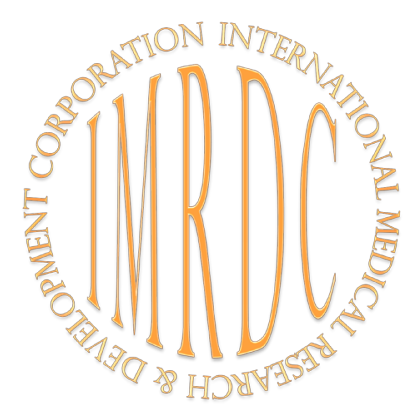

\title{
Creativity as a Determinant of Intraspecific Aggressive Properties of the Psyche of Homo sapiens
}

\author{
Alexander G. Kruglov, PhD, ScD* \\ Central Research Institute of Radiation Diagnosis \\ Moscow, The Russian Federation
}

\begin{abstract}
A sharp and non-linear qualitative change event, namely, the emergence of creativity in the structure of the psyche of Homo sapiens (HS) about 50,000 years ago, created developmental bifurcations, as a result of which HS attained extraspecific and intraspecific domination. Creativity, a new quality of HS psyche, for the first time in the history of hominids enabled HS to separate the image of the goal (IG) from the reactive behavior, transforming IG into an abstract, symbolic object. The emergence of this creative construct, the ambivalent structure existing in the interpretive environment, created a new need for hominids: a relationship with the virtual product of the psyche, a symbol, which has the signs of objective reality in perception. Thus, a fundamentally unsolvable frustration was created: a frustration caused by the inability to achieve equilibrium relations with the controlling symbolic image. Behavior aimed at satisfying needs and eliminating frustration, accompanied by the ordering and structuring of society, acquires under certain conditions the elements of aggression, transforming into sheer aggression. We believe that in the periods preceding the aggression, there is a deliberate deformation of the psychological image of the enemy, the future victim, resulting in the aggressor perceiving the enemy as lacking in human qualities, those qualities that are the attributes of species identity. In this process, the aggressor's psycho-filters and ethical restrictions are eliminated. We understand intraspecific aggression as the antagonistic contact between the frustration constructs, aimed at reducing the tension caused by frustration.
\end{abstract} (International Journal of Biomedicine. 2017;7(2):150-153.)

Key Words: creativity • symbol • aggression • Homo sapiens

The purpose of this research was to describe the fundamental mechanism by which the intraspecific aggression of Homo sapiens (HS) emerged, resulting from the emergence of new psychic qualities of hominids: creativity and symbolic thinking.

To achieve this goal, we used our previously published materials, ${ }^{(1,2)}$ along with the works of cited authors. ${ }^{(3-6)}$ We view the psyche as a distributed system consisting of functional subsystems, where the emergence of new subsystem, creativity, ${ }^{(1)}$ created conditions for a sharp jump-over to a new quality level of the system as a whole, and changed the functionality of the psyche and its transition into a new class with the possibility of implementing goals at a new level.

The emergent phenomenon, a sharp non-linear enhancement of the psychic qualities of HS, which to

*Corresponding author: Alexander G. Kruglov, PhD, ScD. Central Research Institute of Radiation Diagnosis. Moscow, The Russian Federation.E-mail: krag48@mail.ru various degrees is also noted in other hominids, created a developmental bifurcation, the result of which was that HS gained extraspecific and intraspecific domination. Creativity has given to the HS psyche the possibility of separating IG from reactive behavior, i.e. creating the forms of behavior going beyond utilitarian, vital forms.

The visualized $\mathrm{IG}$, the creative construct (CC) having the initial applied (learning) value, transformed into an abstract image becomes an independent object of perception of HS. ${ }^{(1,2)}$ The system of primary abstract representations, transforming over several generations because the means of fixation were lacking, inevitable becomes more complicated, which increases the levels of uncertainty of the setting, the evaluation criteria, and the information volume. Only an individual tolerant of uncertainty (a conditional priest-divergent) is capable of ordering and systematizing primary mythologies, becoming an intermediary between the population and a chaotic set of representations, structuring them and reducing the level of anxious expectations. Integration of $\mathrm{CC}$ by the 
conditional priest for several generations creates a stable virtual construction, ${ }^{(7)}$ which reflects reality in the form of sensual-specific associations, which we are aware of as real. A conditional leader (a convergent) having another set of characterological qualities, is effective in making managerial decisions and initiating the coordinated vector actions of the population (mainly within the coordinates, which are generally accepted in the population).

The cumulation and combination of behavioral coordinates, transformed into ethical coordinates (a derivative of the conditional priest) with the control signals that initiate the concerted actions (a derivative of the conditional leader), transforms the population into a society. The conditional priest and the leader are symbionts performing this transformation, which does not exclude the competition between them. Without a perceptual source, an applied image (IG-CC) in the past acquires the properties of an independent object, which is projected into visual forms ${ }^{(1)}$ with the qualities of an artistic image. The conditional priest maintains a visualized and verbalized virtual product (CC), filling it with semantic content (so that the population appeals to him in order to obtain uncontested arguments), translating control signals, usurping the feedback channel with CC.

For each generation, the virtual images, passed from their predecessors and accompanied by mythologems, are an objective fragment of the habitat, relations with which have the character of bilateral (direct-inverse) connections. Thus, objective reality is endowed with unaccustomed properties (mental product) through the integration of fragments of objective and virtual reality into a single whole, which determines the place and role of the individual in this coordinate system. The result of the above is the formation of a unified information space with reference points of social and ethical coordinates and fixation of the individual's position in this system, leading to the possibility of predictive assessments, a decrease in the level of anxiety, and an increase in social negentropy. As experience of the divergence between forecasts and results accumulates, the idea of one's own insolvency and defenselessness grows. A derivative of this experience is that the control functions are projected onto an external object with a potential superior to that of HS and that the regulation and control functions are delegated to the channeled priest and leader. Thus, the outwardly projected $\mathrm{CC}$ is transformed through certain phases (1st - an applied phase [training], 2nd - a symbolic image separated from reactive behavior, 3rd - a visualized projection that does not have a perceptual source [artistic image] and receives semantic content) and is endowed with regulatory and control functions in relation to the individual and the society. In other words, the psyche of HS produces $\mathrm{CC}$, the projection of which acquires, for human perception, the properties of objective reality with regulatory and control functions that can initiate both individual and concerted social effects, which are not always deterministic and equivalent to the conditions of objective reality.

Lack of the possibility of feedback relations with the controlling $\mathrm{CC}$ initiated a fundamentally new way for the hominids to communicate: a verbal method, supplemented by address code actions (rituals), behavioral algorithms, the substantive part of which was established by intermediaries (priests), i.e., accumulators, carriers of a new kind of anthropological information, channeled relationships with an external controlling object (CC).

A population that transforms into a society establishes a system of interrelated symbols (ethical coordinates) as a standard coordinate system of representations about the boundaries of what is possible and admissible, acquiring stability and reducing the level of anxiety. The creativity of HS, contributing to the emergence and development of symbolic thinking and the expansion of the boundaries of goal-setting beyond the achievable, ${ }^{(1,2)}$ provided the prerequisites for the emergence of frustrations, removing HS beyond the utilitarian forms of vital behavior, which created the possibility of the ambiguous (interpretative) assessment of target images with the extrapolation of virtual structures into objective reality. That is to say, the new intraspecific need that arose for the first time in the history of hominids, namely, the relationship with the symbolic image (CC) which is perceived to have the signs of objective reality created a situation of unsolvable frustration: the impossibility of achieving equilibrium relations with the controlling virtual product (CC). The need, the most important disposition of the motive, is defined as the state of dissatisfaction of the organism (personality), the deficit of what is required to maintain homeostatic equilibrium. $\mathrm{CC}$, for the considered aspect of HS activity, is the leading link in the conscious regulatory process, purposeful behavior, where IG is the virtual construction: the symbol. Due to the ambivalence of the symbol (image+sense) that exists only within interpretations, ${ }^{(8)}$ the one-to-one isomorphic coordinates of the correspondence of any operational image to the final, reference IG (CC) are excluded, and there are convincing signs of homomorphism, where the complete correspondence to the standard (CC) is not possible, but some degree of similarity is achieved. In other words, if the controlling CC, as a symbol, locates the expected result beyond the achievable one, then it creates a primary, unsolvable frustration, which has an inexhaustible negentropic potential for improving the homomorphic sociopsychological constructions of approaching the ideal, the symbolic IG (CC).

The population, transforming into society (regardless of the scale), forms the mechanisms of regulation and management (hierarchy, construction of intrasystem coordinate principles, etc.) and, by so ordering, increases the measure of social negentropy. One of the significant setup principles is the formation, training, and introduction of a set of characteristics (formal and informal) of conformity and belonging to a particular society (from tattoos to confessional attributes), which are identification identifiers, the information and control signals of the recognition filter (ours - alien). We believe that the addressee of the aggressive behavior, the individual (socium), when passed through the aggressor's recognition filter as "alien," is not subjectively considered as belonging to the same species (in the biological sense) as the aggressor. A deficit model of the opponent's psychotype (the future victim) is formed, with the identification of negative features and differences. The concomitant reduction of the restrictive social structure (responsibility, punishment) as 
it regards the aggressor's behavior toward the addressee is a significant addition, which increased the potential for aggression. Demonstrative examples are interracial relations (until recently), as well as interethnic, religious, and other conflicts. The topic of a special study will be changes in the individual and social psyche and psychology in the periods preceding aggression, forming the installation of systems of recognition filters according to the principle "ours - alien." We believe that the result of this process is the deficit states of the psyche, which include the persistent ideas about intraspecific differences (down to antagonistic) and initiate the aggressive behavior and aggression.

In biology, intraspecific aggressive forms of behavior, which have the species-protect functions, are the basis for optimal sexual selection and uniform dispersal (swarming of bees), that optimize the interspecific relationships and trophic connections within the biocenosis and increase the sustainability and diversity of species. The only exception to this series is HS, whose aggressiveness is a phylogenetically altered program at the genetic level, which reflects the instinct of the struggle for existence. ${ }^{(5)}$ Typical of HS is the development of a negative mental state as the response to the impossibility (difficulty) of meeting certain needs that would eliminate frustration. ${ }^{(9)}$ This state includes the whole spectrum of negative emotions (anger, anxiety, irritability), which increase the level of aggression and the likelihood of aggressive actions. The supposed relative rarity of aggressive clashes between primitive societies of antiquity can be explained by the small size of societies that had territorial resources for extensive growth (or displacement) in directions that did not have the specific population resistance. Another reason could be the similarity (affinity) of the totemic values of geographically close communities, which provided the reasons and methods for non-aggressive contacts and associations. Population growth, reduction of compensatory territories, and competition for resource potential led to direct contacts between societies with differences (up to antagonistic ones) in systems of ethical coordinates.

Zones and periods of uncertainty at the borders of these contacts became the arena of a meeting of societies with a stable social structure and symbols. We consider these situations as a dynamic contact between frustration designs that differ in informative filling of the frustration constructs with the potential for antagonistic relations and the likelihood of aggressive actions. Uncertainty in the contact zone is defined as the variability in the choice of forms of behavior from among a set of alternatives and the absence of clear criteria for optimality and efficiency. That is to say, the frustration construct (we stand in solidarity with L. Berkowitz) ${ }^{(10)}$ does not produce aggression immediately, but demands the creation of appropriate conditions with the possibility of exhausting arguments, maturing the antagonistic relations and choosing in favor of aggression, including (in the final stages) the identification by the "ours-alien" filter, the result of which can initiate aggression (the effect of "attack"(11)). Aggression, under the conditions described, is the result of the antagonistic contact between the frustration designs, the ultimate goal of which is the realization of the expectations that cause frustration, thus reducing frustration tension. In the stage that precedes aggression, the opposite side is endowed with properties deforming the attributes of species identity, with a decrease in the filters of the species protection functions, which in turn leads to the elimination of restrictive psychological and other mechanisms in relation to the opponent (victim). Productive, successful aggression leads to domination by the aggressor and regression (destruction) of the frustration construct of the victim, fixing aggression as a way of reducing the frustration tension.

Having considerably simplified the psychological mechanisms that form intraspecific aggressive behavior and aggression, not limited to the scheme we outlined, we aimed to emphasize the existing consistency and dependence of HS intraspecific aggression and the new function - creativity. In our opinion, these traits of HS exist regardless of historical epochs and social formations. A principled scheme of intraspecific aggression of HS, in our opinion, is not contradictory; it supplements the main statements of a number of theories: theory of evolutionism; theory of motivation; theory of cognition; theory of social learning. ${ }^{(12)}$ In the next paper, we intend to consider the differences between and the interdependence of individual and social forms of intraspecific aggression.

\section{Conclusion}

Intraspecific HS aggression is the final stage of negative development of the specific form of adaptive purposeful behavior and realization of a new function unique to HS: the satisfaction of the need for homeostatic relations with the controlling mental construct, a symbol, which is a frustration construct without the opportunity of solution. The negative result of the adaptive period of contact between frustration constructs, which are transformed into antagonistic constructions (aggressor-adversary), initiates the realization of an aggressive potential, which results in a temporary decrease in the stress caused by frustration. The addressee of aggression is endowed with certain properties that reduce and deform the signs of species identity, which leads to the elimination of the species protection filters, the removal of restrictive psychological and other mechanisms, leading HS aggression beyond the limits of intra- and extraspecific regulators of species sustainability and diversity.

\section{Conflicts of interest}

There are no commercial interests or conflicts of interest to declare.

\section{References}

1. Kruglov AG. Creativity as a Determinant of the Development of Homo Sapiens. International Journal of Biomedicine. 2016;6(4):298-302.

2. Kruglov AG. A Mythologem as a Determinant of GoalDirected Behavior. International Journal of Biomedicine. . 2015;5(4):231-234. 
3. Frazer JG. The Golden Bough: A Study in Magic and Religion. Translated from English. M.: TERRA - Knizhnii Klub; 2001:528 pp. [in Russian].

4. Fromm E. The Anatomy of Human Destructiveness. Translated from English. M.: Respublika; 1994:447 pp. [in Russian].

5. Lorenz K. The so-called evil. Translated from German. M.: Kul'turnaia revoluzia; 2008:616 pp.[in Russian].

6. Klein RG. The human career. Human biological and cultural origins. Chicago: The University of Chicago Press; 1989:524 pp.

7. Jung CG. Archetype and Symbol. Translated from English.
M.: Kanon;1991:330 pp. [in Russian].

8. Losev AF. Essays on ancient symbolism and mythology. M.: Misl; 1993:962 pp. [in Russian].

9. Dollard J, Doob L, Miller N, Mowrer O, Sears R. Frustration and aggression. Published by Yale University press 1974 Ex-library Edition. 209 pages.

10. Berkowitz L. Aggression: causes, consequences and control. SPb.: Praim-EVROZNAK; 2001; 512 pp. [in Russian]. 11. Buss A.H. The psychology of aggression. NY: Wiley; 1961: $307 \mathrm{pp}$.

12. Bandura A. The theory of social learning. SPb.: Evrasiia; 2000,320 pp. [in Russian]. 